\title{
EFECTOS DE LA EXPERIENCIA CLÍNICA EN LAS ACTITUDES DE ESTUDIANTES DE ENFERMERÍA HACIA LAS PERSONAS CON DISCAPACIDAD PSIQUIÁTRICA
}

\author{
EFFECTS OF CLINICAL EXPERIENCE IN THE NURSING STUDENT \\ ATTITUDES TOWARD PEOPLE WITH PSYCHIATRIC DISABILITIES
}

\author{
LORENa Parra López ${ }^{*}$ \\ Néstor Ortiz Rebolledo* \\ Claudia Moya Ahumada ${ }^{* * *}$
}

\begin{abstract}
RESUMEN
En la formación del estudiante de pregrado de Enfermería se hace fundamental la elección de experiencias de aprendizaje significativas. Objetivo: Determinar el efecto de la experiencia clínica en servicios de psiquiatría, en las actitudes de estudiantes de Enfermería hacia las personas con discapacidad psiquiátrica. Material y método: Diseño cuasi experimental de pre y postest. La muestra incluyó un total de 84 estudiantes de Enfermería de tercer año de dos universidades de Santiago de Chile. El total de la muestra completó la "Escala de Actitudes hacia las Personas con Discapacidad" (EAPD) forma G. Se consideró como intervención las estrategias usadas en las asignaturas clínicas de enfermería psiquiátrica en ambas universidades. Se realizó prueba t de student para muestras relacionadas. Resultados: Se encontró diferencia estadísticamente significativa $(\mathrm{p}<0,05)$ en el postest, lo que está relacionado con las experiencias de aprendizaje que consideran el contacto con personas con discapacidad psiquiátrica, en diferentes dispositivos de la red de psiquiatría y el acompañamiento docente directo. Conclusión: Estos hallazgos validan la importancia de la elección de experiencias clínicas, en los currículos de formación de Enfermería, como instancias que disminuyen las actitudes negativas como la estigmatización.
\end{abstract}

Palabras clave: Estigma social, personas con discapacidad, educación en enfermería, enfermería psiquiátrica.

\begin{abstract}
The choice of meaningful learning experiences becomes essential in basic Nursing education programs. The purpose of this study is to determine the effect of clinical experience in psychiatric services in Nursing students' attitudes towards people with mental illnesses. A quasi-experimental design method of pre-test and post-test was used. The sample was made up of 84 third-year-nursing students from two universities in Santiago, Chile. All of the participants completed the Scale of Attitudes Toward Disabled Persons (SADP)-G. The strategies used in subjects of psychiatric nursing at both universities were regarded as intervention. Student $t$ test for related samples was performed. Results: Respondents reported statistically significant difference $(p<0.05)$ in the post test, which is associated with learning experiences that consider contact with people with mental illness in any of the different psychiatry network nodes and direct educational support. Conclusion: Validate the importance

\footnotetext{
* Enfermera, Mag. en Investigación Social y Desarrollo. Docente Facultad de Enfermería, Universidad Andrés Bello. Concepción. Autopista 7100 Talcahuano, Chile. Email: lorena.parra@unab.cl

${ }^{* *}$ Enfermero, Mag. en Enfermería. Docente Universidad de Concepción. Concepción. Chile. Email: nortiz@udec.cl

${ }^{* * *}$ Enfermera-psicóloga. Mag. en Psicología Clínica. Docente Facultad de Ciencias para el Cuidado de la Salud, Universidad San Sebastián, Lota 2465, Santiago, Chile. Email: claudia.moya@uss.cl
} 
of including the clinical experience in the nursing training curriculum as a way of reducing negative attitudes of Nursing students, such as stigmatization, towards people with mental illnesses.

Key words: Social stigma, disabled persons, nursing education, psychiatric nursing.

Fecha recepción: 29/12/15 Fecha aceptación: 14/11/16

\section{INTRODUCCIÓN}

A pesar de los cambios en las políticas de salud mental, los avances neurobiológicos y la sensibilización que se ha generado hacia las personas que sufren alguna discapacidad psiquiátrica, aún persiste cierto temor y desconfianza en ellos (1-3).

Se ha ligado muchas veces la discapacidad psiquiátrica con el descontrol y la agresión, quedando mitos de paradigmas antiguos relacionados a cómo se intervenía a una persona con enfermedad mental. La esquizofrenia es una de las enfermedades a la que se asocia con un alto nivel de peligrosidad y agresión, lo que lleva a tener conductas de evitación y estigmatización con las personas que tienen este tipo de patologías (4), pese a que la investigación científica ha demostrado que tienen menores tasas de delitos violentos que la población general (5). Los medios de comunicación han contribuido a mantener las conductas estigmatizadoras de la población, difundiendo noticias donde se da énfasis a la peligrosidad y la impredecibilidad como característica principal de estas personas. De este modo, se da un tratamiento negativo a la información y se deja de lado el seguimiento auténtico del tema (6), minimizando, a la vez, la real vulnerabilidad de las personas que cumplen criterios de vivir con una enfermedad mental, dado que tienen una probabilidad mayor que la población general de ser víctimas de delito, en una razón de entre 2 a 10 veces más (7).

El estigma ha sido parte de la historia en psiquiatría y puede ser entendido según Go- ffman como un "atributo profundamente desacreditador", ya que las personas adoptan una relación asimétrica, donde una adopta la superioridad y la otra, la contraparte, y ninguna de las dos está dispuesta a establecer un contacto en igualdad de condiciones (8). Por su parte, el estigma autopercibido implica una barrera sociocultural para el acceso a los servicios de salud mental y redundan en una menor experiencia de recuperación (9-10).

A raíz de los avances en la salud pública y de la apertura cultural que supone la política sanitaria actual, hoy se espera que las personas que tienen una enfermedad mental puedan desarrollar su vida insertas en el mundo social. Sin embargo, aún se aprecian actitudes discriminatorias, razón por la cual se necesita conocer cuál es la actitud que se tiene hacia las personas con discapacidad psiquiátrica.

Los estudios demuestran que las personas que tienen un contacto cercano con pacientes con discapacidad psiquiátrica, ya sea a través de la vida familiar, práctica profesional o trabajo, tienen menos conductas de estigmatización, lo que fortalecería la red social, mejorando el tratamiento y pronóstico de los usuarios con discapacidad (11-13). Por otro lado, también existe evidencia que en los equipos de salud existe estigma dentro de los profesionales (14), estimándose, en un estudio nepalés, en un 30\% de los profesionales de la salud (15). Badallo et al. plantean que, a pesar de considerarse un bajo nivel de estigmatización, éste, influye negativamente en el proceso de recuperación de los usuarios (16).

Los estudiantes de Enfermería antes de comenzar la práctica clínica de psiquiatría 
describen emociones de miedo, ansiedad e incertidumbre (17) propias del imaginario social en este tema. El conocimiento que presentan los estudiantes de salud en relación a psiquiatría muestra ignorancia en el saber de esta área de la práctica, en especial en aquellos estudiantes formados en currículos donde la psiquiatría no constituye una asignatura independiente (18).

¿Qué ocurre entonces con los estudiantes de Enfermería?, ¿cuál es la actitud que tienen frente a las personas con discapacidad mental?, ¿qué puede facilitar u obstaculizar el proceso de atención hacia el usuario con discapacidad mental y su familia? y ¿cómo se modifican los preconceptos estigmatizadores durante la formación profesional? Estas son preguntas necesarias para abordar el tema en propiedad y el presente estudio intentó asumir esta tarea.

Durante la formación del profesional de enfermería se hace fundamental la elección de experiencias de aprendizaje significativas, siendo muchas veces los enfermeros y enfermeras los que tienen un papel primordial en interferir en el proceso de estigmatización, tanto de las personas que cursan una enfermedad mental, sus familiares, como de los profesionales, contribuyendo a una mayor inclusión de la persona con discapacidad psiquiátrica en la sociedad (19-20).

La experiencia clínica es un espacio de aprendizaje que permite al estudiante poner en práctica el saber que le permitirá actuar. Es una instancia de enseñanza que se produce a través de comprobar e integrar en la práctica la construcción de una relación significativa con el usuario. Con cada aprendizaje se generan nuevos desafíos y van variando los modelos preconcebidos y la imagen del mundo (21).

Cuando los currículos consideran experiencias de aprendizaje de contacto con personas con discapacidad psíquica y sobre todo cuando ese contacto es prolongado, permitiendo ver la recuperación, influye positivamente en la des-estigmatización de las per- sonas que lo experimentan ${ }^{1}$, por lo tanto, si se promueven experiencias significativas en los estudiantes es posible generar un cambio en las actitudes de ellos hacia la persona con enfermedad mental, aumentando la empatía y disminuyendo los estereotipos (22-23).

El objetivo de este estudio es determinar el efecto de la experiencia clínica en servicios de psiquiatría en las actitudes de estudiantes de Enfermería hacia las personas con discapacidad psiquiátrica.

\section{MATERIAL Y MÉTODO}

Diseño cuantitativo, cuasi experimental de pretest y postest en grupos intactos.

La muestra es no probabilística por conveniencia e incluyó un total de 84 estudiantes de Enfermería de tercer año de dos universidades de Santiago de Chile, distribuidos en dos grupos, universidad "A", 30 estudiantes y universidad "B", 54 estudiantes, que aceptaron participar en el estudio de forma voluntaria, anónima y firmaron el consentimiento informado. El protocolo de Investigación fue aprobado por los comités científicos de ambas universidades.

El total de la muestra completó, en modalidad autoadministrada, la "Escala de Actitudes hacia las Personas con Discapacidad" (EAPD) forma G-desarrollada por Verdugo et al. (24), con la que se midió específicamente la actitud hacía la discapacidad psiquiátrica. Se eligió este instrumento por haber sido utilizado en otros estudios en Chile en población similar y por considerarse un instrumento confiable $(\alpha=0,859)$ (25). El instrumento recoge información sobre antecedentes de contacto con personas con discapacidad psiquiátrica, razones y frecuencia del contacto y 37 preguntas, donde se expresa la

${ }^{1}$ Pozo Gil S. La estigmatizacion de la Esquizofrenia [Tesis de grado de Trabajo social]. [Valladolid]: Universidad de Valladolid; 2016. p 43-50. Disponible en: http://uvadoc.uva. es/bitstream/10324/19476/1/TFG-G\%201879.pdf 
opinión respecto de las personas con discapacidad psíquica, eligiendo entre 6 alternativas tipo Likert desde muy en desacuerdo a muy de acuerdo, asignando un valor de 1 a 6 para cada pregunta, excepto en los ítems que expresan valoración negativa que se codificaron de manera inversa en cuanto a los valores asignados en la escala. Una puntuación cercana a 1 en cada pregunta refleja una actitud positiva ante las personas con discapacidad.

En esta investigación se considera como tratamiento experimental las estrategias usadas en las asignaturas clínicas de enfermería psiquiátrica en ambas universidades. En la Universidad "A" los estudiantes realizan su práctica 1 vez a la semana durante 4 meses, lo que corresponde a un total de 48 horas. Pasan por distintos dispositivos, tanto intra como extramurales, de la red de salud mental, permitiéndoles evaluar a personas con distintos grados de compromiso y recuperación. Además, son acompañados permanentemente por enfermeros con experiencia docente y clínica, lo que significa que han recibido formación de algún tipo en docencia universitaria. En la Universidad "B" la práctica es intensiva durante 2 semanas, con $50 \mathrm{~h}$ en una unidad de corta estadía (atendiendo usuarios en estado agudo de la patología) y son acompañados por enfermeros eminentemente clínicos, lo que significa que son enfermeros que habitualmente se han dedicado a la asistencia directa sin muchas vinculaciones con la docencia.

Las mediciones se efectuaron antes de iniciar la asignatura de enfermería psiquiátrica de sus respectivos planes de estudios, asegurándose de que estos conocimientos no influyeran en la percepción que se tenía de las personas con discapacidad psiquiátrica. La segunda medición se realizó tres semanas después de finalizada la asignatura y la experiencia clínica.

Los datos obtenidos fueron analizados en el programa de análisis estadístico SPSS versión 18.0. Se realizó en un primer momento análisis estadístico de tipo descriptivo (medidas de tendencia central) y de variabilidad (desviación estándar) para las variables estudiadas, obteniendo los baremos correspondientes. Se realizó pruebas de $\mathrm{t}$ de student para muestras relacionadas.

\section{RESULTADOS}

Se evalúo primeramente si los estudiantes de Enfermería del estudio habían tenido contacto previo con personas con discapacidad psiquiátrica y la frecuencia del contacto, no encontrándose diferencias estadísticas significativas en esta variable $(p=0,46)$. Esto indica que ambas muestras son equivalentes en este aspecto y que el contacto previo con personas que presentan discapacidad psiquiátrica no influyó en la actitud hacia ellos.

Los resultados mostraron que no hay diferencias estadísticamente significativas en el pretest de ambos grupos $(\mathrm{p}=0,06)$, lo que indica que ambos grupos presentan una actitud hacia las personas con discapacidad similar, antes de vivenciar las experiencias clínicas de las asignaturas de psiquiatría de sus respectivos currículos (Tabla 1 ).

Tabla 1. Comparación puntajes pretest Escala de actitudes hacia las personas con discapacidad, en estudiantes de Universidades A y B.

\begin{tabular}{lcccc}
\hline Universidad & Media & DE & Prueba t & valor p \\
\hline A & $1,544,107$ & $2,315,385$ & $-1,894$ & 0,06 \\
B & $1,608,835$ & $1,904,860$ & & \\
\hline
\end{tabular}


Al comparar los resultados de los puntajes pre y postest al interior de cada grupo, se encontró diferencia estadística significativa, asociada a un p de 0,016, en la Universidad A, lo que significa que la percepción de los alumnos hacia las personas con discapacidad psíquica se vio influenciada con este tipo de experiencia. La Universidad B no presentó diferencias estadísticamente significativas entre la medición pre y postest, obteniéndose un $\mathrm{p}=0,380$ (Tabla 2).

Tabla 2. Comparación de puntajes entre pre y postest al interior de cada grupo, Escala de actitudes hacia las personas con discapacidad en estudiantes de Universidades A y B.

\begin{tabular}{lcccccc}
\hline \multirow{2}{*}{ Universidad } & \multicolumn{2}{c}{ Pretest } & \multicolumn{2}{c}{ Postest } & \multirow{2}{*}{ Prueba t } & \multirow{2}{*}{ Valor p } \\
\cline { 2 - 5 } & Media & DE & Media & DE & & \\
\hline A & $1,465,000$ & $2,073,499$ & $1,612,667$ & $2,326,746$ & $-2,490$ & 0,016 \\
B & $1,591,429$ & 173,848 & $1,624,630$ & $2,053,411$ & $-0,882$ & 0,380 \\
\hline
\end{tabular}

\section{DISCUSIÓN Y CONCLUSIÓN}

En primer lugar, se destaca el hecho de que ambos grupos hayan presentado en el pretest el mismo nivel de actitud hacia la discapacidad psiquiátrica, revelando quizás el estigma social de base que hay sobre este tema, tal como lo plantea la literatura científica $(1,9$, 26, 27).

Exponer a los estudiantes al contacto con usuarios en servicios de corta estadía y comunitarios de salud mental, se podría considerar una estrategia que cumple con los objetivos curriculares de disminuir el estigma percibido, tal como se observa en otras investigaciones, ya que en ellos se puede apreciar personas en proceso de recuperación e inclusión social $(21,22)$. Del mismo modo el hecho de que esta experiencia transcurra en un tiempo prolongado, permite observar algún grado de recuperación de las personas tratadas e ir variando los modelos preconcebidos en las actitudes, aumentando la empatía y disminuyendo los estereotipos ${ }^{1}$. Esta modalidad de experiencia clínica da al estudiante oportunidad de establecer relaciones significativas con los usuarios, situación que favorece el cuidado y facilita el aprendizaje tal como lo plantean varias teorías de enfermería (28). Por último, el acompañamiento de enfermeros con experiencia docente, es otro aspecto de importancia y diferenciador entre ambas universidades. Las tres estrategias pedagógicas recién planteadas son el aporte más significativo de esta investigación a la educación en enfermería en salud mental.

La ventaja mostrada en la Universidad A radica en la posibilidad de efectuar prácticas clínicas en actividades tanto intra como extracomunitarias, donde los usuarios presentan distintos grados de recuperación, son acompañados por enfermeros con experiencia docente y además tienen la posibilidad de realizar prácticas extendidas en el tiempo para poder visualizar la recuperación del usuario. En cambio, en la Universidad B, las prácticas clínicas se realizaron de manera intensiva, acompañados de enfermeros clínicos del mismo servicio y sólo en dispositivos de personas altamente descompensadas, situación que al parecer explica el estigma percibido por los alumnos al finalizar la práctica.

Los profesionales de salud deben cumplir un rol des-estigmatizador que ayude a modificar las actitudes negativas que tiene la población general, la familia e incluso el propio usuario sobre sí mismo. 
Es importante rescatar en este estudio que, al generar un contacto directo y cercano con los usuarios, se pueden disminuir actitudes estigmatizadoras (29-31) y el tipo de experiencias clínicas psiquiátricas que los alumnos de Enfermería experimentan en su currículo puede influir significativamente en disminuir el estigma que se tiene en esta área.

Frente a la necesidad de acreditación de los programas de formación de Enfermería, se hace necesario buscar las mejores estrategias de aprendizaje y formación, considerando la accesibilidad e inclusión de campos clínicos que permitan experiencias de aprendizaje de calidad (32).

La literatura científica ya demuestra lo inconveniente de transverzalizar la psiquiatría supeditándola o incluyéndola como unidades de otras asignaturas (18). En efecto, son múltiples las tareas administrativas y económicas que se juegan en la consecución de campos clínicos para las carreras de la salud, pero si se concibe a la docencia en salud como una estrategia para la transformación social, quizás se debiera considerar como punto principal el tipo de experiencia que los futuros profesionales tienen en este ámbito.

En relación a las limitaciones del estudio, se considera que, si bien la metodología permite alcanzar los objetivos planteados en el estudio, sería necesario identificar qué factores influyen en mayor o menor medida en las actitudes de los estudiantes frente a la discapacidad psiquiátrica. Por otro lado, sería interesante incorporar otras variables que muestren si existen características psicológicas que requieran cambios culturales.

\section{REFERENCIAS}

1. Stuart H. Fighting the stigma caused by mental disorders: past perspectives, present activities, and future directions. World Psychiatry 2008; 7(3): 185-8.
2. Peluso ETP, Blay SL. Public stigma and schizophrenia in São Paulo city. Rev Bras Psiquiatr. 2011; 33(2): 130-136.

3. Kumar D, Kumar P, Singh AR, Bhandari SS. Knowledge and attitude towards mental illness of key informants and general population: a comparative study. Dysphrenia 2012; 3(1): 57-64.

4. Corrigan P, Watson A. The stigma of psychiatric disorders and the gender, ethnicity, and education of the perceiver. Community Ment Health J. 2007; 43(5): 439-58.

5. Heredia M. Esquizofrenia y riesgo de homicidio; una perspectiva europea. La ley penal: revista de derecha penal, procesal y penitenciario. 2014; 110: 7 .

6. Muñoz M, Pérez-Santos E, Crespo M, Guillén A, Izquierdo S. La Enfermedad Mental en los Medios de Comunicación: Un Estudio Empírico en Prensa Escrita, Radio y Televisión. Clínica y Salud [Internet]. 2011 Jul [citado 07 dic 2015]; 22(2): 157-173. Disponible en: http://scielo.isciii.es/scielo.php?script $=$ sci_arttext\&pi$\mathrm{d}=$ S1130-52742011000200005\&lng=e

7. Maniglio R. Severe mental illness and criminal victimization: A systematic review. Acta Psychiatr Scand 2009; 119(3): 18091.

8. Goffman E. Estigma: La identidad deteriorada. Madrid: Amorrortu editores; 1963. p. 172.

9. Campo-Arias A, Oviedo HC, Herazo E. Estigma: barrera de acceso a servicios en salud mental. Rev Colomb de Psiquiatr. 2014; 43(3): 162-7.

10. Garay M, Pousa V, Pérez L. La relación entre la percepción subjetiva del funcionamiento cognitivo y el autoestigma con la experiencia de recuperación de las personas con enfermedad mental grave. Rev. Asoc. Esp. Neuropsiq. [Internet]. 2014 [citado 28 dic 2015]; 34(123): 459-475. Disponible en: http://scielo. isciii.es/scielo.php?script $=$ sci_arttext\&pid=S0211-57352014000300002\&ln- 
g=es. http://dx.doi.org/10.4321/S021157352014000300002.

11. Zárate C, Ceballos M, Contardo M, Florenzano R. Influencia de dos factores en la percepción hacia los enfermos mentales; contacto cercano y educación en salud. Rev. chil. neuro-psiquiatr. 2006; 44(3): 205-214.

12. Polo MT, Fernández C, Díaz C. Estudio de las actitudes de estudiantes de Ciencias Sociales y Psicología: relevancia de la información y contacto con personas discapacitadas. Univ. Psychol. 2011; 10(1): 113-123.

13. Moreno FJ, Rodríguez I, Saldaña D, Aguilera A. Actitudes ante la discapacidad en el alumnado universitario matriculado en materias afines. Revista Iberoamericana de Educación. 2006; 40(5): 1-12.

14. Thornicroft G, Rose D, Kassam A. Discrimination in health care against people with mental illness. Int Rev Psychiatry. 2007; 19(2): 113-22.

15. Adhikari S, Pradhan SN, Sharma SC. Experiencing stigma: Nepalese perspectives. Kathmandu Univ Med J [Internet]. 2008 [citado 07 dic 2015]; 6(24): 458-65. Disponible en http://www.ncbi.nlm.nih.gov/ pubmed/19483426

16. Badallo A, García-Arias S, Yélamos L. La situación de estigmatización en el entorno de un recurso de rehabilitación psicosocial. Rev. Asoc. Esp. Neuropsiq. [Internet]. 2013 Dic [citado 28 dic 2015]; 33(120): 737-747. Disponible en: http://scielo. isciii.es/scielo.php?script $=$ sci_arttext\&pid=S0211-57352013000400005\&ln$\mathrm{g}=$ es. http://dx.doi.org/10.4321/S021157352013000400005

17. Barboza M, Galetto N, González O, Pérez $\mathrm{N}$, Solís M. Estados emocionales de estudiantes de Enfermería en la práctica en el hospital psiquiátrico. Visión de Enfermería Actualizada. 2011; 7(28): 26-29.

18. Gulati P, Das S, Chavan BS. Impact of psychiatry training on attitude of medical students toward mental illness and psychiatry. Ind. J Psychiatry. 2014; 56(3): 271-7.

19. Mahto RK, Verma PK, Verma AN, Singh AR, Chaudhury S, Shantna K. Students' perception about mental illness. Ind. Psychiatry J. 2009; 18(2): 92-6.

20. Ochoa S, Martínez F, Ribas M, García-Franco M, López E, Villellas R. et al. Estudio cualitativo sobre la autopercepción del estigma social en personas con esquizofrenia. Rev. Asoc. Esp. Neuropsiq. [Internet]. 2011 Sep [citado 17 dic 2015 ]; 31(3): 477-489. Disponible en: http:// scielo.isciii.es/scielo.php?script=sci_arttext\&pid=S0211-57352011000300006\&lng=es. http://dx.doi.org/10.4321/S021157352011000300006.

21. Chawla JM, Balhara YP, Sagar R, Shivaprakash Undergraduate medical students' attitude toward psychiatry: A cross-sectional study. Indian J Psychiatry. 2012; 54(1):37-40.

22. Walters K, Raven P, Rosenthal J, Russell J, Humphrey C, Buszewicz M. Teaching undergraduate psychiatry in primary care: the impact on student learning and attitudes. Med Educ. 2007; 41(1): 100-108.

23. Yang L, Lo G, WonPat-Borja A, Singla D, Link B, Phillips M. Effects of labeling and interpersonal contact upon attitudes towards schizophrenia: implications for reducing mental illness stigma in urban China. Soc Psychiatry Psychiatr Epidemiol [Internet]. 2012 [citado 17 dic 2015] 47(9): 1459-73. Disponible en: http:// www.ncbi.nlm.nih.gov/pmc/articles/ PMC3697873/

24. Verdugo M, Arias B, Jenaro C. Actitudes hacia las personas con minusvalía. Madrid, España: Instituto Nacional de Asuntos Sociales; 1994.

25. Mella S, González L. Actitudes de los estudiantes de la salud de la Universidad de Chile hacia las personas con discapacidad psiquiátrica. Rev. Chil. Ter. Ocup. [Internet] 2007 [citado 17 dic 2015] 7: 1-14. Disponible en: http://www.revista- 
terapiaocupacional.uchile.cl/index.php/ RTO/article/viewFile/42/51

26. López M, Laviana M, Fernández L, López A, Rodríguez A, Aparicio A. The struggle against the stigma and discrimination in mental health: A complex strategy based on available data. Rev. Asoc. Esp. Neuropsiq. [Internet]. 2008 [citado 17 dic 2015]; 28(1): 48-83. Disponible en: http://scielo. isciii.es/scielo.php?script=sci_arttext\&pid=S0211-57352008000100004\&ln$\mathrm{g}=\mathrm{es}$

27. Mascayano F, Lips W, Mena C, Manchego C. Estigma hacia los trastornos mentales: características e intervenciones. Salud Ment [Internet]. 2015 Feb [citado 17 dic 2015]; 38(1): 53-58 Disponible en: http://www.scielo.org. $\mathrm{mx} / \mathrm{scielo}$.php? script=sci_arttext\&pi$\mathrm{d}=$ S0185-33252015000100008\&lng=es

28. Marriner A, Raile M. Modelos y Teorías en Enfermería. 7a edición. Barcelona, España: Elsevier Mosby; 2011.816 p.
29. Schulze B. Stigma and mental health professionals: A review of the evidence on an intricate relationship. Int Rev Psychiatry 2007; 19(2): 137-155.

30. Lauber C, Nordt C, Braunschweig C, Rössler W. Do mental health professionals stigmatize their patients? Acta Psychiatr Scand. 2006; 429(Suppl): 51-59.

31. Oliveira S, Carolino L, Paiva A. Programa Saúde Mental Sem Estigma: Efeitos de Estratégias Diretas e Indiretas nas Atitudes Estigmatizantes. Revista Portuguesa de Enfermagem de Saúde Mental [Internet]. 2012 Dic [citado 17 dic 2015]; (8): 30-37. Disponible en: http://www.scielo. mec.pt/scielo.php?script=sci_arttext\&pi$\mathrm{d}=$ S1647-21602012000200005\&lng $=\mathrm{pt}$

32. Jara P, Behn V, Ortiz N, Valenzuela S. Enfermería en Chile. En: Lucas-Breda K. Nursing and Globalization in the Americas: A Critical Perspective. New York, Baywood Publishing. 2009. 55-95. 